\title{
Delayed Presentation of an Unusual Transpharyngeal Penetrating Neck Injury by a Wooden stick: A Case Report
}

\author{
S Grover, DNB, M Chaudhry*, MS \\ Department of Orthopaedics, *Department of Ophthalmology, \\ Maharishi Markandeshwar Institute of Medical Sciences and Research, Haryana, India
}

\begin{abstract}
Penetrating neck trauma is a horrifying injury. Patients may present with sudden death, or life threatening injuries such as catastrophic haemorrhage, major vessel injury, injuries to the respiratory or digestive tract, neurological deficits, or bony injuries of the cervical spine. Other less life threatening symptoms may be associated with such injuries. We present here a case report of a 2-year-old child who sustained a transpharyngeal penetrating neck injury that occurred while playing with a wooden stick. He presented one month later with an abscess in the posterior triangle of the neck.
\end{abstract}

\section{Key Words:}

Transpharyngeal Neck Injury, Delayed Presentation

\section{INTRODUCTION}

Penetrating neck trauma can pose significant diagnostic and therapeutic challenges for emergency physicians. Factors contributing to these problems are complex neck anatomy, proximity of vital structures, and potential for rapid deterioration of airway, vascular, or neurologic injuries ${ }^{1}$. The overall mortality of penetrating neck injuries in the three different anatomical areas (Zones I, II and III) is $9 \%{ }^{2}$.

There is a lack of consensus among trauma surgeons regarding injuries that mandate surgical exploration versus those in which a more conservative approach can be taken ${ }^{3}$. We report a case of a young child presenting one month after suffering a penetrating injury of the neck by a $9 \mathrm{~cm}$ long piece of wooden stick.

\section{CASE REPORT}

A 2 year old boy reported to our department with a 15-day history of fever and swelling on the posterior left side of the neck. On examination, there was a single, diffuse, ovoid swelling approximately $3 \times 2 \times 1.5 \mathrm{~cm}$ in size, located in the posterior triangle of the neck on the left side. Skin overlying the swelling was red, oedematous and shiny. There was no neurovascular compromise, hoarseness of voice or subcutaneous emphysema. Laboratory results revealed an increased total leucocyte count and erythrocyte sedimentation rate. Radiographic examination of the neck (Figure 1) showed that soft tissue planes of neck were obliterated and the prevertebral shadow was increased. The trachea was deviated to the left. CT scan (Figure 2) revealed a linear shadow extending from right side of the pharynx, diagonally across the neck to the left side and ending about $1 \mathrm{~cm}$ beyond the spinous process of the $\mathrm{C} 6$ vertebra; this was reported by the radiologist as an 'artefact'.

A provisional diagnosis of abscess due to foreign body in neck was made and the patient was taken for incision and drainage. Following a difficult endotracheal intubation (with a $3.5 \mathrm{~mm}$ cuffed endotracheal tube) due to laryngopharyngeal oedema, general anaesthesia was initiated. Approximately $10 \mathrm{ml}$ of purulent material was drained from the abscess. Then, a wooden stick, $9 \mathrm{~cm}$ long and $7 \mathrm{~mm}$ thick, was dissected free from the surrounding granulation tissue and removed (Figure 3). Postoperatively, the patient was prescribed a 7-day course of broad spectrum antibiotics. The fever subsided within two days of removal of the foreign object. Similarly, haematological parameters settled down by the third postoperative day.

On retrospective questioning, the patient's mother gave a history that the patient sustained an injury one month prior while playing with a wooden stick. According to her, the child was trying to put the stick in his mouth and lost his balance over uneven ground and fell on the stick. At that time, she tried to remove the stick, but could only remove a part of it as the stick broke in the child's mouth. As the rest of the stick was not visible in the mouth and apparently did not cause any discomfort to the child, the patient as well as his mother did not consider this incident to be of any significance.

The patient was followed up for a period of one year following the surgery. There was no recurrence of infection. Follow-up MRI scan of the patient was performed at one 


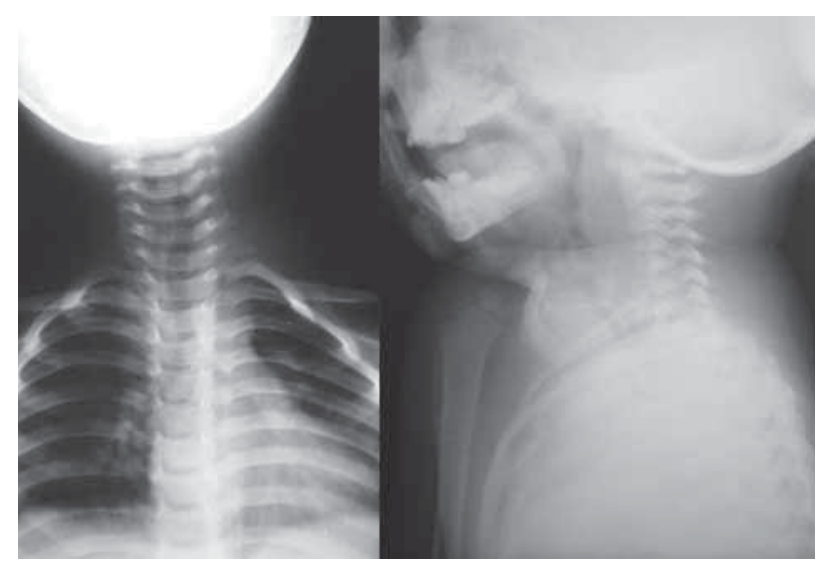

Fig. 1: Radiographs of cervical spine showing increased prevertebral shadow.

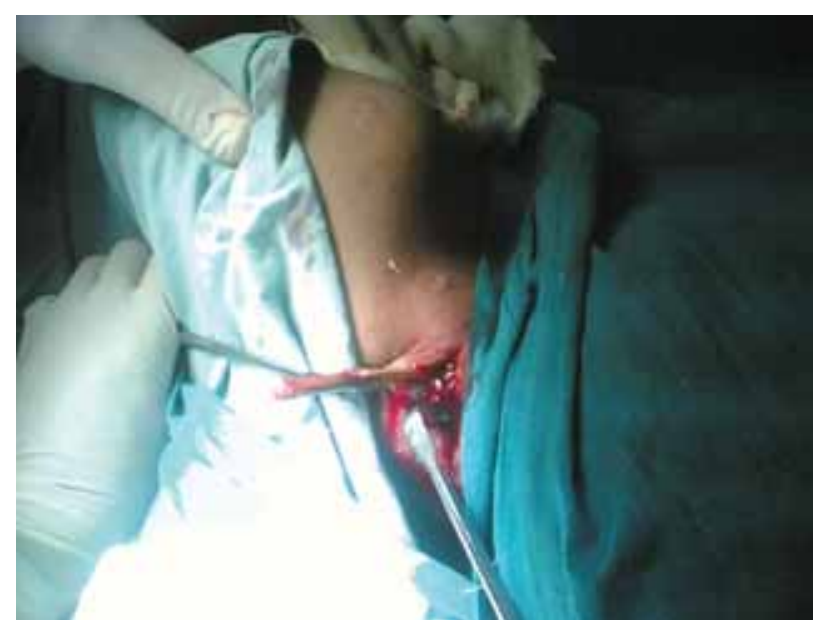

Fig. 3: The foreign body $-9 \mathrm{~cm} \times 7 \mathrm{~mm}$ wooden stick - being removed from postero-lateral aspect of neck.

year post-surgery. No remnant of the foreign body was seen and all tissues had healed well. Clinically, the patient had no symptoms or signs of any obstruction due to fibrosis.

\section{DISCUSSION}

Penetrating neck trauma may pose life threatening consequences, either at the time of injury, while transporting the patient to the hospital or even at a later date if the injury goes unnoticed. Such delayed presentations are, however, rare $^{4}$.

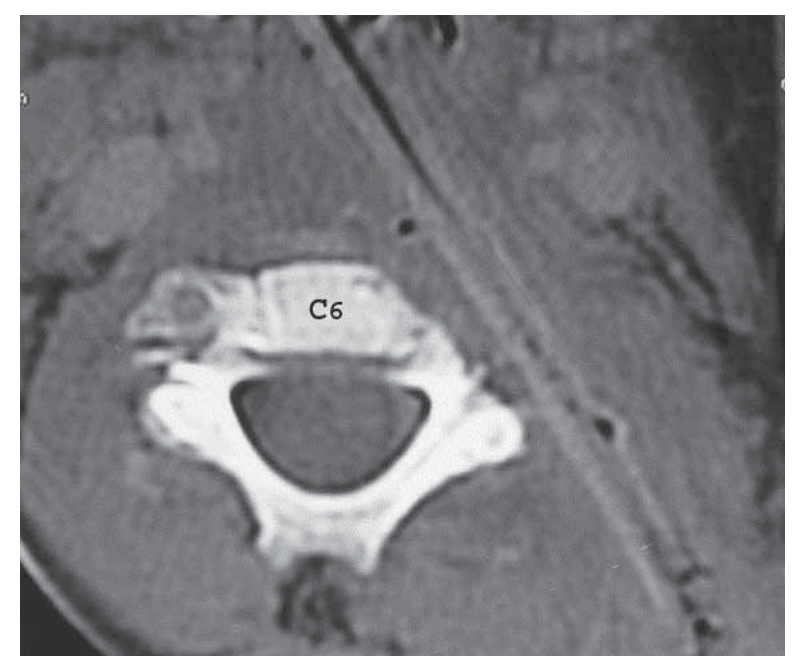

Fig. 2: CT Scan image showing a linear shadow of foreign body across the neck at the level of C6 vertebra.

The neck may be divided into three zones using anatomic landmarks ${ }^{2}$. Zone $\mathrm{I}$ is the horizontal area between the clavicle, suprasternal notch and cricoid cartilage. The proximal common carotid, vertebral, and subclavian arteries, trachea, oesophagus, thoracic duct, and thymus are located in this zone. Zone II is the area between cricoid cartilage and the angle of the mandible, and contains the internal and external carotid arteries, jugular veins, pharynx, larynx, oesophagus, recurrent laryngeal nerve, spinal cord, trachea, and the thyroid, and parathyroid glands. Zone III is the area that lies between the angle of mandible and the base of skull, containing the carotid and vertebral arteries and the uppermost segments of the jugular veins.

Children are most vulnerable to penetrating neck injuries, as they are prone to trauma while playing unconventional outdoor games. In the present case, the injury was in Zone II. Fortunately, none of the vital structures in this zone were damaged even with presentation one month after injury. As any retained foreign body can produce retropharyngeal abscess as a late complication, it should be removed electively, if not as an emergency procedure ${ }^{5}$.

Treating children can be quite challenging for a clinician. They usually present with peculiar symptoms, which may not point towards a particular disease or injury. Injuries in the neck region are inherently dangerous. If in doubt, a detailed history from the parents and thorough scrutiny may yield vital clues to the diagnosis of the pathology, which may be missed during initial evaluation. Hence, a level of suspicion about an injury in a young patient and re-thinking the history and initial investigations may be rewarding in reaching a final diagnosis. 


\section{REFERENCES}

1. Kendall JL, Anglin D, Demetriades D. Penetrating neck trauma. Emerg Med Clin North Am. 1998; 16(1): 85-105.

2. Hersam G, Barker P, Bowley DM, Boffard KD. The management of penetrating Neck injuries. Int Surg 2001; 86(2): 82-9.

3. Demetriades D, Theodorou D, Cornwell E 3rd, Weaver F, Yellin A, Velmahos G, et al. Penetrating injuries of the neck in patients in stable condition. Physical examination, angiography, or color flow Doppler imaging. Arch Surg 1995; 130(9): 971-5.

4. Lee ST. A delayed unusual presentation of a penetrating foreign body. Singapore Med J 1992; 33(3): 304-5.

5. Yickles JM. Retropharyngeal abscess complicating a neck wound: a case report. J Laryngol Otol 1988; 102(6): 552-3. 